\title{
CMB anisotropy science: a review
}

\author{
Anthony Challinor \\ Institute of Astronomy and Kavli Institute for Cosmology Cambridge, Madingley Road, \\ Cambridge, CB3 0HA, U.K. \\ DAMTP, Centre for Mathematical Sciences, Wilberforce Road, Cambridge, CB3 0WA, U.K. \\ email: a.d.challinor@ast.cam.ac.uk
}

\begin{abstract}
The cosmic microwave background (CMB) provides us with our most direct observational window to the early universe. Observations of the temperature and polarization anisotropies in the CMB have played a critical role in defining the now-standard cosmological model. In this contribution we review some of the basics of CMB science, highlighting the role of observations made with ground-based and balloon-borne Antarctic telescopes. Most of the ingredients of the standard cosmological model are poorly understood in terms of fundamental physics. We discuss how current and future CMB observations can address some of these issues, focusing on two directly relevant for Antarctic programmes: searching for gravitational waves from inflation via $B$-mode polarization, and mapping dark matter through CMB lensing.
\end{abstract}

Keywords. cosmology: cosmic microwave background

\section{Introduction}

It is now twenty years since the landmark discovery of fluctuations in the temperature of the cosmic microwave background radiation by the COBE satellite (Smoot et al. 1992). Over the intervening period, a now-standard cosmological model has emerged. The CMB fluctuations have been pivotal in putting this model on a firm observational footing (though many of its key ingredients continue to defy explanation in fundamental physics), and in measuring its parameters to a level of precision that is unprecedented in cosmology. While the game-changer in this field has undoubtedly been the full-sky measurements from the WMAP satellite, observations of the CMB from Antarctica have played an important role in this development and have achieved a number of significant 'firsts'. These include precision measurements of spatial flatness and the detection and, now, characterisation of linear polarization of the CMB.

This symposium covers a broad range of astrophysics so the purpose of this review is to set the scence for the other more specialised CMB contributions that follow. We begin by reviewing some of the basics of CMB science and the remarkable achievements made through observations of the CMB temperature and polarization fluctuations, highlighting the role of measurements made from Antarctica. Experiments in Antarctica are also very much at the cutting edge of future programmes seeking to address some of the questions raised by the standard cosmological model. Space limits us to discuss in detail only two of the main science goals of these experiments: the quest for gravitational waves and CMB lensing. For more complete recent reviews of CMB science, see Challinor \& Peiris (2009) and $\mathrm{Hu}(2008)$.

\section{The CMB and the standard cosmological model}

In the standard cosmological model, named $\Lambda \mathrm{CDM}$, the universe is well described on large scales by a spatially-flat, homogeneous and isotropic background metric with small 
fluctuations at the $10^{-5}$ level. The universe has evolved from a hot, dense phase during which matter and radiation were in thermal equilibrium at sufficiently early time. The CMB is the thermal relic radiation from this early phase and its existence is a cornerstone of the hot big bang model. The CMB radiation has now cooled to a temperature of $2.725 \mathrm{~K}$ but retains an almost perfect blackbody spectrum. Baryons and leptons make up $4.5 \%$ of the current energy density and cold dark matter (CDM; hypothesised matter with essentially only gravitational interactions and negligible thermal velocities) $22 \%$. The remaining $73 \%$ is in the form of dark energy and drives the current accelerated expansion. Dark energy is not understood at all at a physical level but phenomenologically behaves like a smoothly distributed fluid with equation of state close to $p=-\rho$, as for a cosmological constant $\Lambda$.

The flatness and large-scale smoothness of the universe are neatly explained by a hypothesised period of quasi-exponential expansion - cosmic inflation - in the early universe. During a period of only $10^{-32} \mathrm{~s}$, the universe expanded in size by at least 60 e-folds. Inflation is not understood at a fundamental level, but it can be realised in simple models by a scalar field $\phi$ evolving slowly over a flat part of its self-interaction potential $V(\phi)$. A compelling feature of inflation is that it naturally provides a causal mechanism for generating primordial curvature perturbations and gravitational waves with nearly scale-free power spectra. Small-scale quantum fluctuations in light scalar fields, and the spacetime metric, are stretched beyond the Hubble radius during inflation to appear later as classical, long-wavelength cosmological perturbations that seed the growth of largescale structure. In simple models (e.g. $V(\phi) \propto \phi^{2}$ ) inflation at energies $E_{\text {inf }} \sim 10^{16} \mathrm{GeV}$ reproduces the observed level of perturbations.

\subsection{Temperature anisotropies}

The CMB carries an imprint of the primordial perturbations via small temperature anisotropies at the $O\left(10^{-5}\right)$ level. The universe became transparent to CMB photons around the time of recombination, when atomic hydrogen (and helium) first formed. This defines a last-scattering surface centred on our current location, and spatial fluctuations in the CMB energy density, bulk velocity and gravitational potential over this surface project to give temperature anisotropies in the CMB. In more detail, for curvature perturbations, the fractional anisotropy $\Theta(\hat{\mathbf{n}})$ along a direction $\hat{\mathbf{n}}$ at time $t_{0}$ is given approximately by

$$
\Theta(\hat{\mathbf{n}})=\Theta_{0}+\psi-\hat{\mathbf{n}} \cdot \mathbf{v}_{b}+\int_{t_{*}}^{t_{0}}(\dot{\psi}+\dot{\phi}) d t .
$$

Here, $\Theta_{0}$ is the fractional fluctuation in the CMB temperature on the last-scattering surface (time $t_{*}$ ), $\mathbf{v}_{b}$ is the baryon peculiar velocity, and $\psi$ and $\phi$ are the gravitational potentials $(\phi=\psi$ in general relativity when non-relativistic matter is dominant). Each term has a simple physical interpretation: we see the intrinsic temperature fluctuation $\Theta_{0}$, modified by the gravitational-redshifting effect of the potential $\psi$ and the Doppler shift from scattering off moving matter. The final integrated Sachs-Wolfe term in Eq. (2.1) involves the integral of the time derivatives of $\psi$ and $\phi$; if a potential well is getting shallower in time (as happens during dark-energy domination), photons receive a net blueshift in crossing the well and the CMB appears hotter. In practice, around $10 \%$ of photons were re-scattered after the universe reionized which, on all but the largest scales, reduces the primary anisotropies sourced around recombination by a factor $e^{-\tau}$, where $\tau \approx 0.1$ is the Thomson optical depth.

The small amplitude of the temperature anisotropies means they can be calculated very accurately with linear perturbation theory. The fluctuations on the last-scattering 


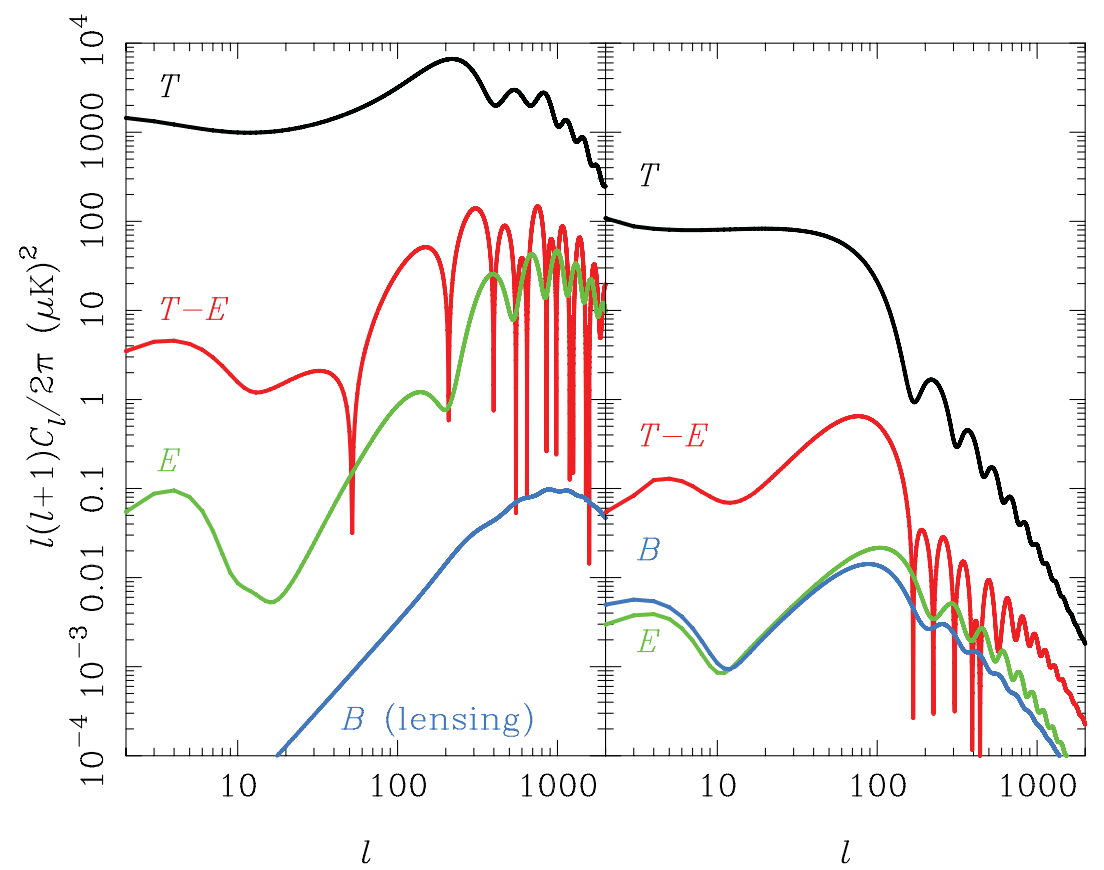

Figure 1. Temperature (black), $E$-mode (green), $B$-mode (blue) and $T E$ cross-correlation (red) CMB power spectra from curvature perturbations (left) and gravitational waves (right) for a tensor-to-scalar ratio $r=0.24$. The $B$-mode spectrum induced by weak gravitational lensing is also shown in the left-hand panel (blue).

surface are therefore a linearly-processed version of the nearly scale-free primordial perturbation. On scales large compared to the Hubble radius at last-scattering, only gravity is important but on smaller scales the acoustic physics of the primordial plasma and photon diffusion dominate. Gravity-driven infall will tend to enhance a positive density perturbation, but this is resisted by photon pressure setting up acoustic oscillations in the plasma. The sine and cosine-like modes of oscillation extrapolate back to decaying and constant modes at early times. Inflation is democratic, putting equal power into each mode at generation, but any decaying mode is totally negligible by the time the acoustic oscillations begin. This process leaves only cosine-like oscillations in the plasma, so that oscillations on all scales start off in phase. However, different scales oscillate at different frequencies and scales which have reached extrema of their oscillations by lastscattering have enhanced power in the anisotropies on the corresponding angular scales. In this way, the sound horizon $r_{s}\left(t_{*}\right)$, i.e. the (comoving) distance a sound wave can have propagated by time $t_{*}$, introduces a preferred length scale to the fluctuations. It is a fortunate coincidence that the corresponding angular scale $r_{s} / d_{A}$ (where $d_{A}$ is the angular-diameter distance back to last-scattering) is around $1^{\circ}$ and so straightforward to observe at frequencies around $100 \mathrm{GHz}$ where the CMB is brightest.

Figure 1 shows the predicted angular power spectrum, $C_{l}^{T}$, from inflationary curvature perturbations. The power spectrum is the variance of the multipoles $\Theta_{l m}$ in a sphericalharmonic expansion $\Theta(\hat{\mathbf{n}})=\sum_{l m} \Theta_{l m} Y_{l m}(\hat{\mathbf{n}})$. The multipole index $l$ corresponds roughly to anisotropies at scale $180^{\circ} / l$. The plateau in $C_{l}$ on large scales is from the combination of primary anisotropies on scales large enough to be unaffected by acoustic processing, and from the integrated-Sachs-Wolfe effect from late-time decay of the gravitational potentials. On intermediate scales, we have acoustic peaks. Finally, on smaller scales 


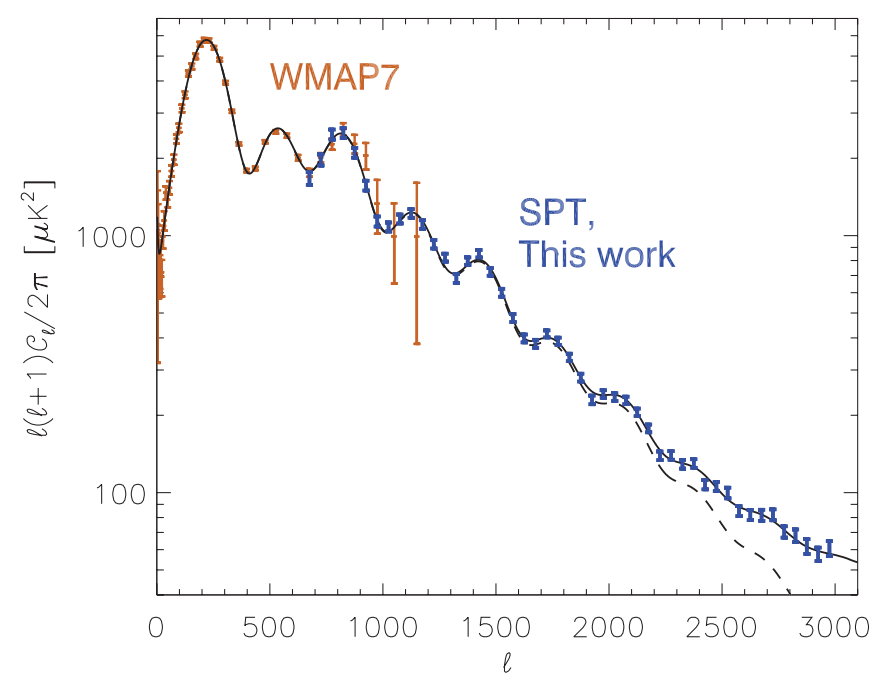

Figure 2. Measurements of the temperature power spectrum from WMAP (orange; Larson et al. 2011) and $790 \mathrm{deg}^{2}$ of the SPT 150-GHz survey (blue; Keisler et al. 2011). Also shown are the CMB spectrum (dashed) and the total spectrum (CMB and extragalactic foregrounds; solid) in the best-fitting $\Lambda$ CDM model. Reproduced with permission from Keisler et al. (2011).

the power decays rapidly. This damping tail is sourced by perturbations on scales small enough that photons had time to diffuse out of overdensities by last-scattering, thus damping out the acoustic oscillations. This process imprints another scale, the diffusion scale, into the CMB.

The picture outlined above is spectacularly confirmed by measurements of the temperature anisotropy. Theory only allows us to predict the statistical properties of the primordial perturbation. In simple models of inflation, the statistics are Gaussian, and so fully characterised by their power spectrum. Primordial Gaussianity is borne out by careful measurements of the statistics of the CMB anisotropies. For this reason, the main focus of observational CMB research for the past 20 years has been to obtain precise estimates of the $\mathrm{CMB}$ angular power spectrum and to confront these against theoretical models. An example of such measurements from the WMAP satellite (Larson et al. 2011) and the South Pole Telescope (SPT; Keisler et al. 2011) is shown in Fig. 2. Nine acoustic peaks have now been measured and the SPT measurements thoroughly characterise the damping tail. The error bars on the power spectrum include the effects of instrument noise and cosmic/sample variance - at each $l$ we estimate the power spectrum from the empirical variance from a sample of only $(2 l+1) f_{\text {sky }}$ independent quantities (where $f_{\text {sky }}$ is the sky fraction covered by the survey). The Planck survey (The Planck Collaboration 2006), which is expected to report its first CMB results in early 2013, will improve considerably the statistical power of the CMB power spectrum measurements between $l=500$ where WMAP becomes noise limited, and $l=2000$ where Planck's poorer resolution and sensitivity loses out to SPT despite the greatly extended sky coverage. Beyond $l \sim 2000$, measurements start to be contaminated by the unresolved background of extra-Galactic sources at all frequencies.

Given that the physics of the CMB is so well understood, the standard cosmological model can be tested very precisely and its parameters determined to high precision (see e.g. Komatsu et al. 2011; Keisler et al. 2011). Here, for brevity, we can highlight only three examples. 
Primordial power spectrum: This affects the overall morphology of the CMB power spectrum. Parameterising the spectrum of primordial curvature perturbations as a powerlaw, $\mathcal{P}_{\mathcal{R}}(k) \propto k^{n_{s}-1}$, WMAP constrains $n_{s}=0.963 \pm 0.014$ (assuming no contribution to the CMB from gravitational waves; Larson et al. 2011). This is beautifully consistent with the inflationary prediction of a nearly scale-free spectrum $\left(n_{s}=1\right)$. A departure from scale-invariance is detected at almost the $3 \sigma$ level and provides important constraints on the dynamics of inflation [i.e. the slope and curvature of $V(\phi)]$. The combination of the $n_{s}$ constraint and upper limits on the gravitational wave power spectrum (see Section 3) already rule out several simple inflation models.

Matter densities: The relative heights of the acoustic peaks are influenced by the physical densities of baryons and CDM. For example, increasing the baryon fraction adds inertia but not pressure support to the plasma, reducing the bulk modulus. This increases the overdensity at the midpoint of the acoustic oscillations boosting the compressional peaks (1st, 3rd etc.). Precise measurements of the physical baryon density, $\Omega_{b} h^{2}=0.02258 \pm 0.00056$ (Larson et al. 2011), have been made via this route, nicely consistent with constraints from big-bang nucleosynthesis. This $3 \%$ precision should improve to around $1 \%$ with Planck data. Similarly, the CDM density is measured to be $\Omega_{c} h^{2}=0.1109 \pm 0.0056$. This provides inescapable evidence of the need for non-baryonic dark matter independently of other lines of reasoning such as the clustering and internal kinematics of galaxies.

Curvature: The angular scale of the acoustic peaks $r_{s} / d_{A}$ is now very precisely measured. In standard models, the matter densities determined from the relative peak heights fully determine $r_{s}$, thus allowing an accurate measurement of the angular-diameter distance to last scattering. This distance is very sensitive to spatial curvature through its geometrical focusing effect, but this can always be compensated by altering the radial distance to last-scattering (through the Hubble constant $H_{0}$ or, equivalently, the dark energy density). This leads to a geometric degeneracy whereby models with the same physical densities at high redshift, the same primordial power spectrum, and the same angular-diameter distance to last-scattering give almost identical angular power spectra. (An example is given later in Fig. 4.) The degeneracy can be broken by adding other astrophysical distance measures such as the Hubble constant, the angular-diameter distance at lower redshift (inferred from the relic of the baryon acoustic oscillations - BAO - in the clustering of galaxies; Eisenstein et al. 2005) or the luminosity distance inferred from supernovae. An early, important example of determining curvature via this route was from the 1998 flight of BOOMERanG (de Bernardis et al. 2000). By precisely characterising the first acoustic peak, the team were able to establish that space was flat at the $10 \%$ level. More recent measurements are consistent with flatness at the $0.5 \%$ level (Komatsu et al. 2011), strongly supporting one of the main predictions of inflationary cosmology.

\subsection{Polarization}

The other key CMB observable is polarization. Thomson scattering of unpolarized radiation with a quadrupole $(l=2)$ anisotropy in its total intensity generates linear polarization. The relevant epoch for the generation of polarization in the CMB is around recombination since at early times scattering is too efficient to allow a significant quadrupole to grow, while after recombination scatterings are very rare (until the universe reionizes). The expected linear polarization from curvature perturbations has an r.m.s. of $5 \mu \mathrm{K}$.

Linear polarization can be described by two Stokes parameters $Q$ and $U$. These depend on a choice of basis and measure the difference in intensity transmitted by linear polarizers aligned with the basis directions $(Q)$ or at $45^{\circ}$ to them $(U)$. While Stokes parameters provide a local, operational definition of polarization, their coordinate dependence makes 

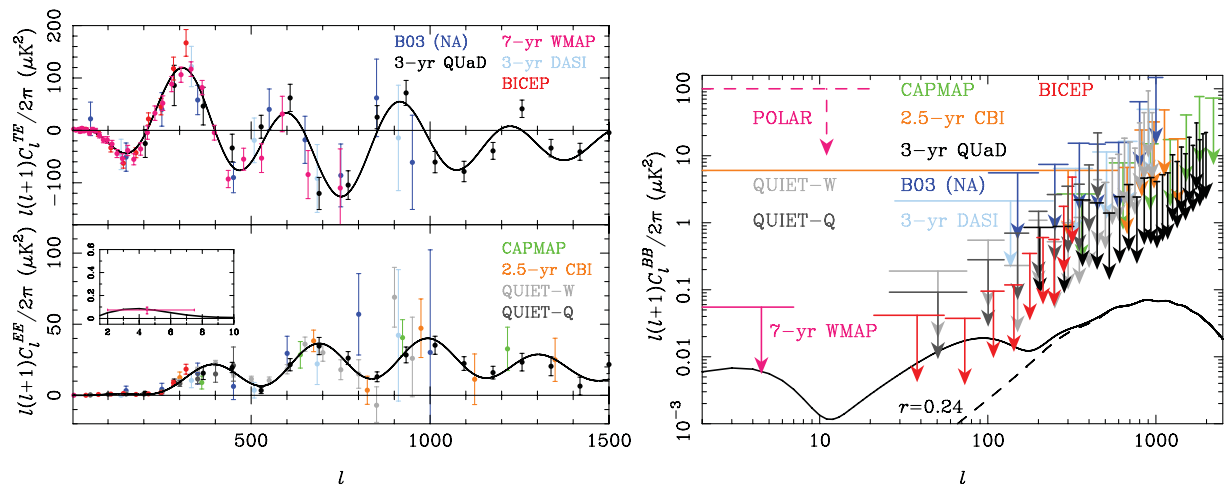

Figure 3. Left: current measurements of the polarization power spectra $T E$ (top) and $E E$ (bottom) from WMAP7 (magenta; Larson et al. 2011), QUaD (black; Brown et al. 2009), BOOMERanG (blue; Piacentini et al. 2006; Montroy et al. 2006), DASI (cyan; Leitch et al. 2005), CAPMAP (green; Bischoff et al. 2008), CBI (orange; Sievers et al. 2007), BICEP (red; Chiang et al. 2010) and QUIET $W$-band (light grey; QUIET Collaboration 2012) and QUIET Q-band (dark grey; QUIET Collaboration 2011). The lines are $\Lambda$ CDM fits to temperature and polarization data. Right: current $95 \%$ upper limits on the $B B$ power spectrum including the constraint from POLAR (dashed cyan; Keating et al. 2001). The dashed line is the contribution from gravitational waves for $r=0.24$, the $95 \%$ upper limit from fits to the temperature and $E$-mode polarization data from WMAP7 combined with BAO and $H_{0}$ measurements (Komatsu et al. 2011), and the solid line includes the contribution from gravitational lensing.

them rather inconvenient for cosmological interpretation. Instead, linear polarization can be described in terms of two scalar fields, $E$ and $B$ (Seljak \& Zaldarriaga 1997; Kamionkowski et al. 1997). The Stokes parameters are properly the components in an orthonormal basis of a rank-2 symmetric, trace-free tensor which can be expressed in term of second derivatives of $E$ and $B$ (neglecting sky curvature for simplicity, and using Cartesian coordinates):

$$
\left(\begin{array}{cc}
Q & U \\
U & -Q
\end{array}\right) \propto\left(\partial_{i} \partial_{j}-\frac{1}{2} \delta_{i j} \nabla^{2}\right) E+\epsilon_{k(i} \partial_{j)} \partial_{k} B .
$$

This is analogous to decomposing a vector field into a gradient part $(E)$ and a divergencefree curl part $(B)$. Note that $E$ and $B$ are non-local in $Q$ and $U$.

The $E$-modes are scalars under parity but $B$-modes are pseudo-scalar. In the absence of parity-violating physics, the two fields must be uncorrelated. This leaves three nonzero polarization power spectra: the $E$ - and $B$-mode auto-correlations $C_{l}^{E}$ and $C_{l}^{B}$, and the cross-correlation $C_{l}^{T E}$ between $E$ and the temperature anisotropies. The predicted angular power spectra for inflationary curvature perturbations are shown in the left-hand panel of Fig. 1. The main points to note are as follows: (i) polarization is a small signal; (ii) $E$-mode polarization peaks on smaller scales than the temperature, since it relies on diffusion in small-scale modes for its generation; (iii) the acoustic peaks in $C_{l}^{E}$ are at the troughs of $C_{l}^{T}$ since the temperature quadrupole derives mostly from the plasma bulk velocity which vanishes when the density is at an extremum; (iv) there is a 'bump' in the polarization on large scales generated by re-scattering once the universe reionizes; and (v) by symmetry, curvature perturbations cannot generate $B$-mode polarization except through second-order processes such as gravitational lensing (see Section 4). This last point makes $B$-modes a potentially powerful probe of gravitational waves; see Section 3 .

Observations of $\mathrm{CMB}$ polarization are not yet as advanced as for the temperature anisotropies. Current power spectrum measurements are shown in Fig. 3. Antarctic 
experiments have played a very significant role, including the first detection of CMB polarization by the DASI interferometer in 2002 (Kovac et al. 2002), and the current best characterisation of the spectra by QUaD (Brown et al. 2009) and BICEP (Chiang et al. 2010). The measurements are in excellent agreement with expectations based on the temperature power spectrum, providing an important consistency test. Moreover, through large-angle $E$-modes, WMAP measures the optical depth to reionization to be $\tau=0.088 \pm 0.015$ (Larson et al. 2011), providing an important integral constraint on astrophysical models of reionization. Future $E$-mode polarization measurements will tighten parameter constraints over those from the temperature anisotropies, particularly in non-standard models, and extend the angular range that can be reliably probed before foregrounds dominate. However, the real excitement over polarization is the prospect of detecting the signature of gravitational waves via $B$-modes and exploiting $B$-modes induced by weak lensing.

We end this section by emphasising that, despite the triumph of the standard cosmological model in fitting essentially all cosmological data (with just six parameters), the model raises several big questions. Did inflation happen? What is the nature of dark matter? Why is the universe accelerating? In the following sections, we review how ongoing and future CMB observations will help answer some of these questions.

\section{Gravitational waves and $B$-mode polarization}

Inflation naturally predicts the production of a stochastic background of primordial gravitational waves accompanying the primordial density perturbation (Starobinskii 1979). The spectrum of gravitational waves depends only on the expansion rate during inflation. Since this is nearly constant during slow-roll inflation, with only a slow decrease, the primordial spectrum $\mathcal{P}_{h}(k)$ should be well approximated by a power-law with a slightly red spectrum. As the Friedmann equation relates the expansion rate directly to the energy density during inflation, a measurement of the gravitational wave power gives directly the energy density and hence the energy scale $E_{\text {inf }}$ during inflation. It is conventional to express the amplitude of $\mathcal{P}_{h}(k)$ in terms of its ratio to the power spectrum of curvature perturbations $\mathcal{P}_{\mathcal{R}}(k)$ at a cosmologically-relevant scale (often $k_{0}=0.002 \mathrm{Mpc}^{-1}$ ). This tensor-to-scalar ratio $r$ is related to $E_{\text {inf }}$ by

$$
r=8 \times 10^{-3}\left(E_{\text {inf }} / 10^{16} \mathrm{GeV}\right)^{4},
$$

where we have taken the scalar amplitude $\mathcal{P}_{\mathcal{R}}\left(k_{0}\right)=2.36 \times 10^{-9}$. Note that $r \sim 10^{-2}$ for inflation occurring around the GUT scale, $E_{\text {inf }} \sim 10^{16} \mathrm{GeV}$.

Gravitational waves damp away due to the expansion of the universe when their wavelength is smaller than the Hubble radius. The best prospect for detection is therefore via the $\mathrm{CMB}$ which is sensitive to early times (after last-scattering) and large scales. Gravitational waves generate CMB temperature anisotropies due to the integrated effect of the anisotropic expansion they induce along the line of sight; see Fig. 1. However, the signal is limited to large angular scales, $l<60$, corresponding to gravitational waves with wavelengths larger than the Hubble radius at last-scattering. On such scales, chance upwards fluctuations in the temperature anisotropies from curvature perturbations due to cosmic variance limit our ability to measure $r$. In the optimistic scenario that all other cosmological parameters are know, cosmic variance gives a $1 \sigma$ error on $r$ of 0.07 from the temperature anisotropies alone. In practice, degeneracies make the CMB-only limit a little worse: e.g. $r<0.21$ (at 95\% confidence) from WMAP7+SPT (Keisler et al. 2011), improving on $r<0.36$ from WMAP7 alone (Komatsu et al. 2011). Gravitational waves also leave an imprint in the linear polarization of the CMB. Significantly, they 
generate $E$ - and $B$-modes with roughly equal power, unlike curvature perturbations which only generate $B$-modes at second order through gravitational lensing. In principle, $B$-mode measurements of $r$ can do much better than inferences from the temperature or $E$-mode polarization since the former is only limited by the cosmic variance of the lensinduced $B$-modes $\dagger$. The problem is that the $B$-mode signal is very small (see Fig. 1 ); the limit $r<0.24$ implies that the r.m.s. from gravitational waves is less than $200 \mathrm{nK}$. The measurement therefore requires exquisite sensitivity and control of systematic effects to maintain polarization purity, and careful rejection of polarized emission from our Galaxy.

Current upper limits on the $B$-mode power spectrum are shown in Fig. 3 . The best constraints over nearly the full range of scales come from BICEP (Chiang et al. 2010) at degree scales and QUaD (Brown et al. 2009) on smaller scales. The BICEP constraint of $r<0.73$ (95\% confidence) is not yet competitive with that from the temperature anisotropies, although it is rather less model dependent. There are two main scales to attempt detection of $B$-modes from gravitational waves: $l<10$ where the signal is generated by scattering at reionization, and $l \sim 100$ where the signal from scattering around recombination peaks. The reionization signal needs a nearly full-sky survey and so broad frequency coverage to remove Galactic emission which is dominant over most of the sky. The best near-term constraints on these scales will come from Planck with forecasts indicating $r<0.05$ may be achievable (Efstathiou \& Gratton 2009). The signal from recombination can be constrained by targeting clean, connected regions of the sky (typically around $1000 \mathrm{deg}^{2}$ ) in areas of low Galactic emission. By good fortune, one of the cleanest such regions is accessible from Antarctica. A series of results from BICEP's successors, BICEP2, Keck and POLAR (see contributions from Pryke and Kuo in this volume), as well as the balloon-borne SPIDER (Filippini et al. 2010) and several experiments based in Atacama, are eagerly anticipated over the next five years. These should push down the errors on $r$ to around 0.01 . This is an interesting target for inflationary physics since the signal from a large class of simple models - "large-field" such as monomial potentials - would be detectable. Looking further ahead, the constraint on $r$ could plausibly be improved to the $10^{-4}-10^{-3}$ level with a future polarization satellite.

\section{Weak gravitational lensing of the CMB}

The fluctuations in the temperature of the CMB are mostly imprinted at the epoch of last scattering. However, CMB photons undergo small gravitational deflections due to the clumpy distribution of matter (weak gravitational lensing) as they propagate from lastscattering to the present epoch. The r.m.s. deflection is only 2.7 arcmin but is coherent over several degrees. The lensing effect is similar to seeing the CMB fluctuations from the last-scattering surface through patterned glass, and subtly distorts their statistics. With telescope resolution of a few arcminutes or better, these distortions can be detected and used to reconstruct the lensing deflection. This opens up a new cosmological probe of structure formation at epochs and scales that are difficult to access with more direct probes (such as galaxy clustering). Lensing is an emerging field in observational CMB research and results from the SPT are at the forefront of this.

Weak lensing has several important effects on the CMB; see Lewis \& Challinor (2006) for a detailed review. Magnification and demagnification of the acoustic-scale features leads to a smoothing of the acoustic peaks, reaching the $10 \%$ level at $l>2000$ in

$\dagger$ In principle, the cosmic variance from lensing can even be removed by "delensing" the observed $Q$ and $U$ maps with a reconstruction of the lensing deflection field (Seljak \& Hirata 2004). The latter can be obtained from the CMB itself with high-resolution polarization observations; see Section 4. 

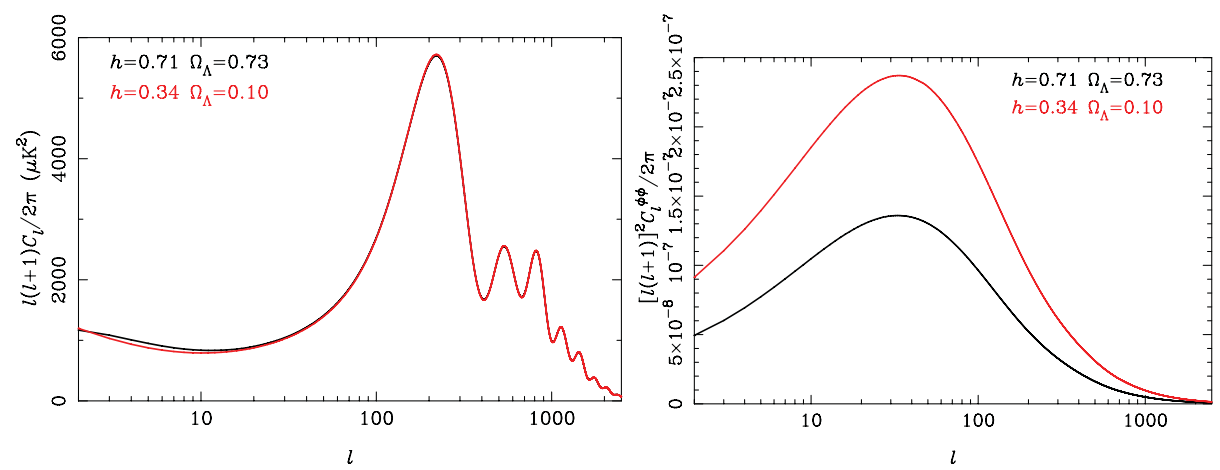

Figure 4. Breaking the angular-diameter distance degeneracy with CMB lensing. The unlensed (and unobservable!) temperature power spectra (left) for the standard $\Lambda \mathrm{CDM}$ model (black) and a closed model with low $H_{0}$ to match the angular scale of the acoustic peaks (red) are very nearly degenerate. The degeneracy is broken in the power spectrum of the lensing deflection angle (right) since matter is more clustered at late times in the model with low $H_{0}$.

temperature, and rather larger in E-mode polarization. On smaller scales, for which the unlensed CMB is very smooth, lensing generates small-scale power that dominates the primary anisotropies for $l>4000$. The lens remapping moves around the polarization amplitude while preserving the direction, generating $B$-modes from the primary $E$-modes with an almost white spectrum for $l \ll 1000$; see Fig. 1. As noted in Section 3, this will become an important source of confusion for CMB searches for gravitational waves. Finally, lensing introduces four-point non-Gaussianity with a very specific and predictable shape from which the full angular power spectrum $l(l+1) C_{l}^{\phi \phi}$ of the lensing deflections can be reconstructed $\ddagger$. Through these lensing effects, the CMB is sensitive to parameters that have degenerate effects in the primary anisotropies. For example, Fig. 4 compares the unlensed temperature power spectra and the deflection power spectra for the standard $\Lambda$ CDM model and a closed model with low $H_{0}$. These models lie along the geometric degeneracy of the unlensed CMB power spectra. However, the deflection spectra are quite different since matter is more clustered at late times in the low- $H_{0}$ model. Other parameters that benefit similarly from lensing information include sub-eV neutrino masses and early dark energy.

The first measurements of the deflection power spectrum from the four-point function of the temperature anisotropies have recently been reported by the Atacama Cosmology Telescope (ACT; Das et al. 2011) and SPT (van Engelen et al. 2012). These are in excellent agreement with expectations for the standard $\Lambda$ CDM model; see Fig. 5. The current SPT measurements constitute a $6.3 \sigma$ detection but they are from only $590 \mathrm{deg}^{2}$ of sky. The significance can be expected to increase several-fold with the analysis of the full $2500 \mathrm{deg}^{2}$ survey, similar to what should be achieved with Planck. Already, combining the SPT lens reconstruction with the temperature power spectrum from WMAP breaks the geometric degeneracy in $\Lambda$ CDM models with curvature giving a significant detection of dark energy from the CMB alone; see the right-hand panel of Fig. 5. The degeneracy is also broken by the effect of lensing on the high- $l$ temperature power spectrum itself, as measured, for example, by SPT (Keisler et al. 2011).

Lens reconstruction from the CMB temperature suffers from statistical noise due to chance correlations in the unlensed CMB that mimic the effect of lensing. This is such that temperature reconstructions will never give cosmic-variance-limited measurements of the

$\ddagger$ In linear theory, the deflection is the gradient of the lensing potential $\phi$. The lensing potential is an integrated measure of the gravitational potential along the line of sight. 

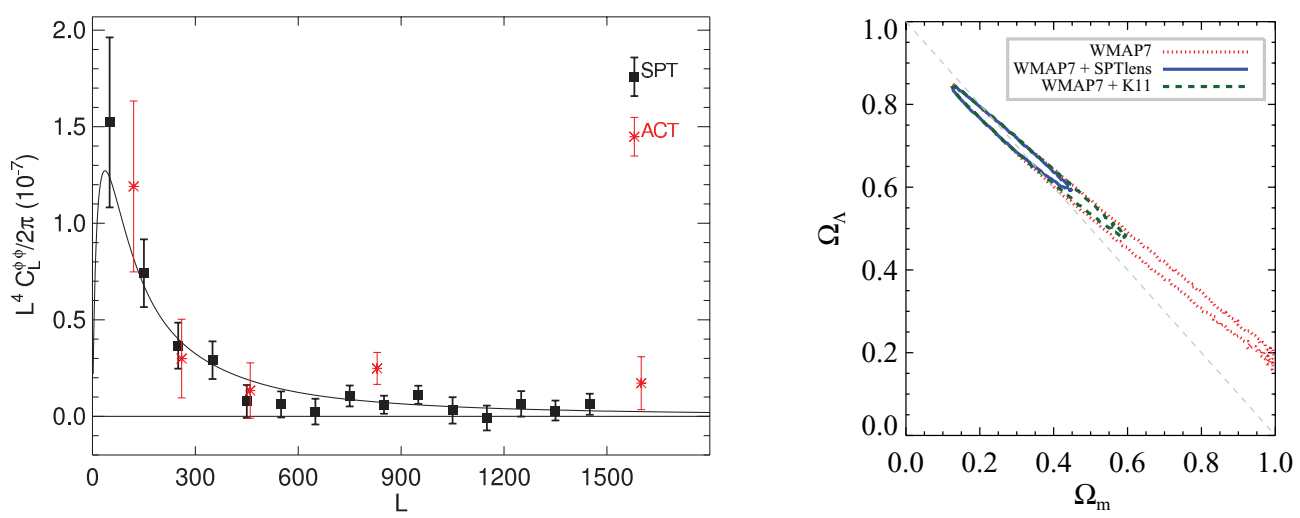

Figure 5. Left: Current measurements of the lensing deflection power spectrum from SPT (black; van Engelen et al. 2012) and ACT (red; Das et al. 2011). The solid line is a $\Lambda$ CDM fit to CMB temperature and polarization data, but not to the lensing data. Right: $95 \%$ confidence regions in the $\Omega_{\Lambda}-\Omega_{m}$ plane for $\Lambda \mathrm{CDM}$ models with curvature. The geometric degeneracy is evident in the WMAP7-alone constraints (red), but the tail of low- $H_{0}$ closed models is cut-off by the higher-resolution SPT data which is sensitive to the lensing effect in the temperature power spectrum (green; Keisler et al. 2011). Even tighter constraints are obtained by combining the SPT lens reconstruction from the left-hand figure with the WMAP7 data (blue). Figure reproduced with permission from van Engelen et al. (2012).

deflection power spectrum for multipoles $l>100$. Polarization measurements are very helpful here (Hu \& Okamoto 2002), since they intrinsically have more small-scale power and the $B$-mode of polarization is not confused by primary anisotropies. In principle, polarization can provide cosmic-variance limited reconstructions to multipoles $l \approx 500$, i.e. on all scales where linear theory applies. For this reason, lens reconstruction from polarization is an important science goal for the polarization upgrades to the SPT (McMahon et al. 2009) and ACT (Niemack et al. 2010), as well as proposed successors to the Planck satellite (Bock et al. 2008, 2009; The COrE Collaboration 2011).

\section{Outlook}

The future of CMB observations lies on several fronts. Precise polarization measurements on large scales will greatly improve limits on the stochastic background of gravitational waves predicted from inflation in the early universe. Wide-area, high-resolution temperature and polarization measurements will allow precise reconstruction of the gravitational-lensing effect in the $\mathrm{CMB}$ and provide a new window to the large-scale clustering of matter around redshift two. In addition, arcminute-scale observations will provide catalogues of thousands of galaxy clusters over a broad redshift range and with well-understood selection functions, and measure the Doppler signatures from the bulk flows of matter in the post-reionization universe. The cluster catalogues will be used to probe the growth of structure and evolution of the volume element to high redshift. These programmes address directly many of the outstanding issues raised by the standard cosmological model, such as the physics of inflation and the cause of the current accelerated expansion.

\section{References}

Bischoff C. et al., 2008, ApJ, 684, 771

Bock J. et al., 2009, ArXiv e-prints 0906.1188 
Bock J. et al., 2008, ArXiv e-prints 0805.4207

Brown M. L. et al., 2009, ApJ, 705, 978

Challinor A. \& Peiris H., 2009, in American Institute of Physics Conference Series, Vol. 1132, Novello M., Perez S., eds., pp. 86-140

Chiang H. C. et al., 2010, ApJ, 711, 1123

Das S. et al., 2011, Phys. Rev. Lett., 107, 021301

de Bernardis P. et al., 2000, Nature, 404, 955

Efstathiou G. \& Gratton S., 2009, JCAP, 6, 11

Eisenstein D. J. et al., 2005, ApJ, 633, 560

Filippini J. P. et al., 2010, in SPIE Conference Series, Vol. 7741, Holland W. S., Zmuidzinas J., eds., pp. $77411 \mathrm{~N}-77411 \mathrm{~N}-12$

Hu W., 2008, ArXiv e-prints 0802.3688

Hu W. \& Okamoto T., 2002, ApJ, 574, 566

Kamionkowski M., Kosowsky A., \& Stebbins A., 1997, Phys. Rev. Lett., 78, 2058

Keating B. G., O’Dell C. W., de Oliveira-Costa A., Klawikowski S., Stebor N., Piccirillo L., Tegmark M., \& Timbie P. T., 2001, ApJ. Lett., 560, L1

Keisler R. et al., 2011, ApJ, 743, 28

Komatsu E. et al., 2011, ApJS, 192, 18

Kovac J. M., Leitch E. M., Pryke C., Carlstrom J. E., Halverson N. W., \& Holzapfel W. L., 2002, Nature, 420, 772

Larson D. et al., 2011, ApJS, 192, 16

Leitch E. M., Kovac J. M., Halverson N. W., Carlstrom J. E., Pryke C., Smith M. W. E., 2005, ApJ, 624, 10

Lewis A. \& Challinor A., 2006, Phys. Rep., 429, 1

McMahon J. J. et al., 2009, in American Institute of Physics Conference Series, Vol. 1185, Young B., Cabrera B., Miller A., eds., pp. 511-514

Montroy T. E. et al., 2006, ApJ, 647, 813

Niemack M. D. et al., 2010, in SPIE Conference Series, Vol. 7741, Holland W. S., Zmuidzinas J., eds., pp. 77411S-77411S-21

Piacentini F. et al., 2006, ApJ, 647, 833

QUIET Collaboration, 2012, ArXiv e-prints 1207.5034

QUIET Collaboration, 2011, ApJ, 741, 111

Seljak U. \& Hirata C. M., 2004, Phys. Rev. D, 69, 043005

Seljak U. \& Zaldarriaga M., 1997, Phys. Rev. Lett., 78, 2054

Sievers J. L. et al., 2007, ApJ, 660, 976

Smoot G. F. et al., 1992, ApJ. Lett., 396, L1

Starobinskii A. A., 1979, JETP Lett., 30, 682

The COrE Collaboration, 2011, ArXiv e-prints 1102.2181

The Planck Collaboration, 2006, ArXiv e-prints astro-ph/0604069

van Engelen A. et al., 2012, ApJ, 756, 142 